\title{
CONTRIBUCION AL CONOCIMIENTO DE LOS MIXOMICETOS DEL PARQUE NACIONAL LAGUNAS DE ZEMPOALA
}

\author{
Gema Galindo-Flores \\ Laura Hernandez-Cuevas \\ Mercedes Rodriguez-Palma \\ $Y$ \\ Arturo Estrada-Torres \\ Centro de Investigación en Ciencias Biológicas,
Universidad Autónoma de Tlaxcala
Apdo. Postal 63-389, México 28000, D.F.
}

\section{RESUMEN}

Se revisaron 91 ejemplares de mixomicetos recolectados en el Parque Nacional Lagunas de Zempoala, encontrándose 20 especies pertenecientes a 13 géneros. Los órdenes mejor representados en la zona fueron los Trichiales con 7 especies y los Liceales con 6 . Se realizó un análisis preliminar sobre las preferencias por los sustratos que ocupan las especies y su distribución mundial. De acuerdo con dicho análisis los mixomicetos encontrados se pueden agrupar en tres categorlas: especies generalistas, especies lignícolas y especies predominantemente lignicolas, pero que eventualmente pueden ocupar otros sustratos. Con excepción de Cribraria purpurea y Dictydium mirabile que sólo se conocen de zonas de clima templado y Ceratiomyxa fruticulosa, Arcyria cinerea, Fuligo septica y Comatricha nigra que tienen distribución cosmopolita, el resto de las especies estudiadas tienen amplia distribución sin poder considerarse como verdaderamente cosmopolitas.

\section{ABSTRACT}

Twenty species of Myxomycetes collected in the Lagunas de Zempoala National Park were identified. The best represented orders were the Trichiales with 7 species and the Liceales with 6 species. A preliminary analysis of substrate's preferences of the species suggests the existence of three categories: generalistic species, lignicole species and predominantly lignicole species but eventually growing over other substrates. Analysis of world distribution of these species shows that all of them have a fairly large range of distribution, with exception of Cribraria purpurea and Dictydium mirabile, which only have been reported from temperate regions and Ceratiomyxa fruticulosa, Arcyria cinerea, Fuligo septica and Comatricha nigra that are truly cosmopolitan species.

\section{INTRODUCCION}

Por sus caracteristicas, los parques nacionales son áreas importantes para la conservación de nuestros recursos, de ahí el interés de conocer lo que en ellos existe (Toledo, 1988). Entre los más cercanos a la Ciudad de México se encuentra el Parque Nacional Lagunas de Zempoala, cuya vegetación se encuentra representada por bosques de Pinus-Abies, asociación que en nuestro país se encuentra confinada a las zonas montañosas con climas 
fríos y una elevada humedad ambiental (Rzedowski, 1978). A pesar de la importancia de dicha zona, hasta el momento no se ha realizado un estudio micologico formal en ella y solamente se han dado a conocer algunas especies de hongos en forma aislada (Mendiola y Guzmán, 1973; Frutis et al., 1985; López et al., 1985). Las únicas especies de mixomicetos citadas para esta región son hasta el momento Dictydium cancellatum (Batsch.) Macbr. (Villarreal, 1990) y D. mirabile (Rost.) Meylan (Hernández-Cuevas et al., 1991).

La escasez de estudios de mixomicetos de áreas protegidas mexicanas y la importancia de las Lagunas de Zempoala como un área dedicada a la consenvación de los recursos naturales, motivaron la realización del presente estudio.

\section{AREA DE ESTUDIO}

El Parque Nacional Lagunas de Zempoala se encuentra ubicado entre los $19^{\circ} 02^{\prime} 26^{\prime \prime}$ y los $19^{\circ} 05^{\prime} 00^{\prime \prime}$ de latitud norte y los 99 $18^{\prime} 15^{\prime \prime}$ y los 99 $19^{\prime} 16^{\prime \prime}$ de longitud oeste, a una altitud comprendida entre los 2500 y los 3000 m s.n.m., situándose en los límites de los estados de México y Morelos (Fig. 1). El clima predominante es templado subhúmedo, el más húmedo de los subhúmedos, con lluvias en verano, $\left(C\left(w_{2}\right) w\right)$, de acuerdo con la clasificación de Köppen modificada por García (1988).

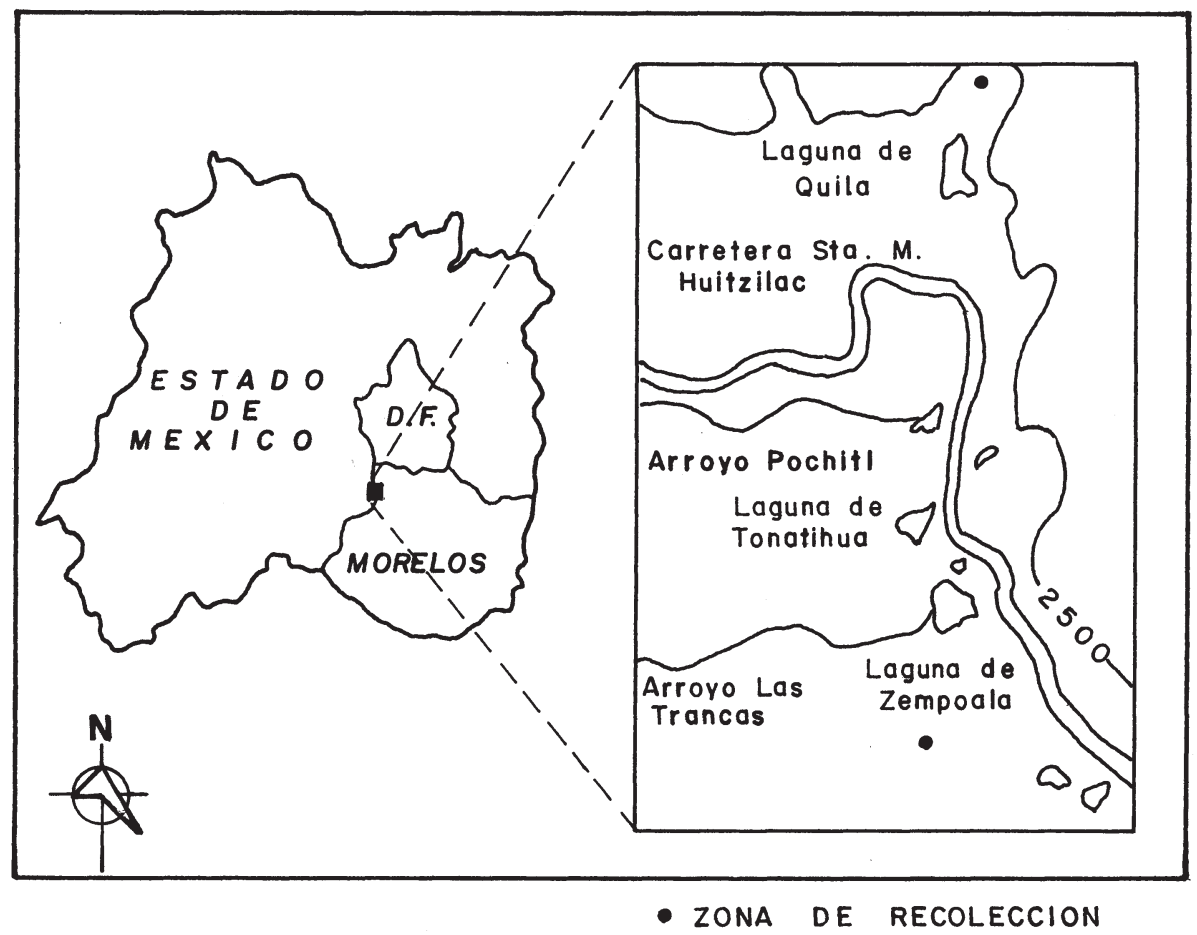

Fig. 1. Localización del área de estudio 


\section{MATERIALES Y METODOS}

Los organismos se recolectaron en las zonas boscosas circundantes a las lagunas de Quila, Estado de México y Zempoala, Morelos. En cada uno de estos sitios se establecieron tres estaciones de muestreo. En la Laguna de Quila, las tres áreas seleccionadas presentaron una vegetación predominante de bosque de Abies, en tanto que en Zempoala los lugares de muestreo se ubicaron en bosques de Pinus, de Pinus-Abies y Abies.

Se realizaron un total de 4 salidas al campo entre mayo y agosto de 1987 . Solamente se recolectaron los mixomicetos que se desarrollaban en troncos de árboles caldos y otros sustratos asociados a los mismos (musgos, liquenes y hongos). El material fue herborizado y determinado con base en sus características macro y microscópicas utilizando las técnicas micológicas rutinarias; las observaciones se efectuaron con lupa y microscopios de campo claro y/o de contraste de fases. La determinación se realizó siguiendo las claves de Martin y Alexopoulos (1969) y Farr (1976, 1981), y consultando las descripciones de Braun y Keller (1976), Keller y Braun (1977), Gómez-Sánchez y Castillo (1981), Trujillo et al. (1986) y Trujillo (1988).

Los colores de las estructuras fueron caracterizados con base en los atlas de Methuen (Kornerup y Wanscher, 1978) y Munsell (U.S. Department of Agriculture, 1975).

La literatura anterior y los trabajos de Welden y Lemke (1961), Welden y Guzmán (1978), Welden et al. (1961), Guzmán (1972, 1983), López et al. (1979, 1981, 1981a, 1981b), Mapes et al. (1981), Martínez-Alfaro et al. (1983), Villarreal (1983, 1990), Chacón y Guzmán (1984), Guzmán y Villarreal (1984) y Pérez-Moreno y Villarreal (1988) fueron utilizados para realizar un análisis preliminar sobre la distribución de las especies en México y su preferencia por sustratos determinados.

Para conocer la distribución mundial y los sustratos en los que se han registrado las especies estudiadas, se realizó una revisión bibliográfica exhaustiva.

El material fue comparado y corroborado con ejemplares de referencia del herbario de la Escuela Nacional de Ciencias Biologicas (ENCB) del Instituto Politécnico Nacional y fue depositado en el mismo herbario con algunos duplicados en la Colección Micológica del Centro de Investigación en Ciencias Biológicas (C.I.C.B.) de la Universidad Autónoma de Tlaxcala.

\section{RESULTADOS}

Se encontraron 20 especies de mixomicetos de las cuales 12 son nuevos registros para el Estado de México y 8 para Morelos. De este conjunto, 7 pertenecen al orden Trichiales, 6 a los Liceales, 3 a los Physarales, 3 a los Stemonitales y 1 a los Ceratiomyxales.

Las especies estudiadas se enlistan a continuación. Las marcadas con (*) representan nuevos registros para el Estado de México y las señaladas con (**) son nuevos registros para Morelos. Para cada una se anexa alguna referencia bibliográfica en la cual se encuentra incluida una descripción detallada basada total o parcialmente en especímenes mexicanos.

Hemitrichia clavata, Physarum viride y Stemonitis flavogenita se describen ampliamente ya que en la literatura revisada no existen descripciones basadas en material mexicano. 


\section{CERATIOMYXOMYCETIDAE}

CERATIOMYXALES

Ceratiomyxaceae

* Ceratiomyxa fruticulosa (Mull.) Macbr.

Descripción: López et al., 1979.

Material estudiado:ESTADODE MEXICO: Hernández-Cuevas 5, 6, 12;Rodríguez-

Palma 24; Vilchis 7. MORELOS: Rojas 3.

\section{MYXOGASTROMYCETIDAE}

LICEALES

Enteridiaceae

* Enteridium lycoperdon (Bull.) Farr

Descripción: Farr, 1976.

Material estudiado: ESTADO DE MEXICO: Rojas 1.

* * Lycogala epidendrum (L.) Farr

Descripción: Farr, 1976.

Material estudiado: ESTADO DE MEXICO: Galindo 13; Rodriguez-Palma 5, 8, 22 ;

Vilchis, 2. MORELOS: Hernández-Cuevas 9; Rodríguez-Palma 21, 26; Vilchis 8, 13.

Tubifera ferruginosa (Batsch.) Gmel.

Descripcion: Trujillo et al., 1986.

Material estudiado: ESTADO DE MEXICO: Galindo 19; Rodriguez-Palma 23.

MORELOS: Galindo 24; Vilchis 15.

Cribrariaceae

* Cribraria purpurea Schrad.

Descripción: Trujillo, 1988.

Material estudiado: ESTADO DE MEXICO: Galindo 5.

** Dictydium cancellatum (Batsch.) Macbr.

Descripción: López et al., 1979.

Material estudiado: ESTADO DE MEXICO: Hernández-Cuevas 1, 15, 24.

MORELOS: Galindo 22; Hernández-Cuevas 17; Rodríguez-Palma 27; Vilchis 9.

D. mirabile (Rost.) Meylan

Descripción: Hernández-Cuevas et al., 1991.

Material estudiado: ESTADO DE MEXICO: Hernández-Cuevas 20.

TRICHIALES

Trichiaceae

*** Arcyria cinerea (Bull.) Pers.

Descripción: Farr, 1976.

Material estudiado: ESTADO DE MEXICO: Vilchis 19. MORELOS: Hernández-

Cuevas 31; Rodriguez-Palma 11. 


\title{
* A. ferruginea Sauter
}

Descripción: Braun y Keller, 1976.

El material de las Lagunas de Zempoala concuerda con la descripción de Martin y Alexopoulos (1969) excepto en la forma de los esporangios ya que en el caso de los especímenes de Zempoala son globosos y de acuerdo con dichos autores deben de ser algo alargados.

Material estudiado: ESTADO DE MEXICO: Hernández-Cuevas 19.

** A. incarnata (Pers.) Pers.

Descripción: López et al., 1979.

Material estudiado: MORELOS: Vilchis 22.

* Hemitrichia clavata (Pers.) Rost.

Descripción: Fructificaciones esporangiadas, escasamente agrupadas, piriformes, de hasta $2.5 \mathrm{~mm}$ de altura total, de color café amarillento brillante (Methuen: 5DE8), con dehiscencia irregular en la parte superior del esporangio y formando una especie de calículo en la madurez; peridio reticulado; estípíte corto, hasta de $0.5 \mathrm{~mm}$ de altura, de color café negruzco (Methuen: 7F8), atenuado hacia la base; hipotalo membranoso, negruzco, común a todos los esporangios; capilicio de color café amarillento brillante (Methuen: 5D8), algunas veces con tonos oliváceos, no muy denso, elástico, ramificado, anastomosado, espiralado, unido firmemente a la base del esporangio, con pocas puntas libres obtusas. Esporas de color café muy pálido (Munsell: 10YR 8/3) en masa, amarillo pálido por la luz transmitida, globosas, de casi lisas a ligeramente papiladas, dando la apariencia de presentar reticulaciones cerradas débilmente marcadas, de 7.0 a $8.0 \mathrm{~mm}$ de diámetro.

Material estudiado: ESTADO DE MEXICO: Galindo 11; Hernández-Cuevas 4; Vilchis 4, 5. MORELOS: Rodriguez-Palma 18; Vilchis 24.

* H. serpula (Scop.) Rost.

Descripción: Gómez-Sánchez y Castillo, 1981.

Material estudiado: ESTADO DE MEXICO: Rodriguez-Palma 3, 7.

*** Trichia decipiens (Pers.) Macbr.

Descripción: Farr, 1976.

Material estudiado: ESTADODE MEXICO: Galindo 1,2, 12; Hernández-Cuevas 2, 13; Rojas 2; Rodriguez-Palma 1; Vilchis 1, 11. MORELOS: Galindo 4, 7, 8; HernándezCuevas 3, 7, 16; Rodriguez-Palma 15, 16, 19, 20, 29; Vilchis 16.

** T. favoginea (Batsch.) Pers.

Descripción: Farr, 1976.

Material estudiado: MORELOS: Hernández-Cuevas 18; Rodríguez-Palma 17;

Vilchis 20.

\author{
PHYSARALES \\ Physaraceae \\ " Fuligo septica (L.) Wiggers
}


Descripción: Farr, 1976.

Material estudiado: MORELOS: Estrada-Torres 2175.

* Physarum nutans Pers.

Descripción: Gómez-Sánchez y Castillo, 1981.

Material estudiado: ESTADO DE MEXICO: Galindo 23; Vilchis 17, 21.

* Ph. viride (Bull.) Pers.

Descripción: Fructificaciones esporangiadas, estipitadas, de 1.5 a $2.0 \mathrm{~mm}$ de altura total, gregarias, subglobosas, de color amarillo verdoso (Methuen: 1A7); peridio membranoso, hialino, con dehiscencia irregular en el ápice y regular formando lóbulos hacia la base; estípite atenuado en el ápice, de amarillo brillante a casi transparente, cubierto con limo de color amarillo limón (Methuen: 1A7); hipotalo membranoso, común a varias fructificaciones, de color rojizo muy obscuro (Munsell: 10YR 2.5/1); capilicio denso, formado por filamentos hialinos, anastomosados, con nodos fusiformes de color verde brillante (Methuen: 1A7). Esporas negras en masa, de color café pálido por la luz transmitida, globosas, de 9.0 a $11.0 \mathrm{~mm}$ de diámetro, equinuladas.

Muy similar a Physarumnutans, con la cual se confunde fácilmente en el campo y de la que se diferencia por el color brillante de los nodos de carbonato de calcio de Ph. viride. Dicho color se pierde fácilmente con el lavado y sólo se conserva en la porción central de los nodos, por lo cual se hace necesaria la revisión microscópica para poder distinguir entre ambas especies.

Material estudiado: ESTADO DE MEXICO: Galindo 16, 18; Hernández-Cuevas agos. 1, 1987; Vilchis 18.

\section{STEMONITOMYCETIDAE}

Stemonitaceae

*** Stemonitis axifera (Bull.) Macbr. Descripción: Farr, 1976.

Material estudiado: MORELOS: Hernández-Cuevas 18; Rodriguez-Palma 17;

Vilchis 10.

** S. flavogenita Jahn.

Descripcion: Cuerpos fructíferos esporangiados, estipitados, de color rojizo muy opaco (Munsell: 2.5 YR2.5/2), cilíndricos, densamente agregados formando fascículos, hasta de $6.2 \mathrm{~mm}$ de altura total; estípite hasta de $2.0 \mathrm{~mm}$ de altura, negro rojizo (Munsell: 10 YR 2.5/ 1), proyectándose dentro del esporangio formando una columnela que termina en el ápice donde frecuentemente forma una expansión membranosa; capilicio formado por una red delicada de filamentos que se originan en la columnela, de color café rojizo muy opaco (Munsell: 10 YR 2.5/1), con numerosas puntas libres y expansiones membranosas; hipotalo membranoso, de color café rojizo a negruzco. Esporas de color café obscuro (Munsell 10 YR 2.5/3) en masa, de color café pálido por la luz transmitida, de (6.0) 7.0 a $8.0(9.0) \mathrm{mm}$ de diámetro, finamente verrucosas.

Los especímenes mexicanos presentan esporas ligeramente más pequeñas que las mencionadaspor Martin y Alexopoulos (1969). 
Material estudiado: MORELOS: Galindo 15; Lorenzana 1.

* Comatricha nigra (Pers.) Schroet.

Descripción: Gómez-Sánchez y Castillo, 1981.

30.

Material estudiado: ESTADO DE MEXICO: Galindo 17; Hernández-Cuevas 25,

Los sustratos ocupados por cada especie se enlistan en el cuadro 1. En el cuadro 2 se incluye la información conocida hasta ahora acerca de la distribución de las especies en la República Mexicana y los tipos de vegetación en los que cada una de ellas han sido encontradas. En el cuadro 3 se hace referencia a los países o regiones geográficas en los que tales especies han sido registradas.

\section{DISCUSION}

Todas las especies de mixomicetos en las Lagunas de Zempoala, crecen directamente sobre madera de duramen, aunque ocho de ellas se encontraron también sobre otros sustratos como musgos ( 6 especies), madera de albura ( 3 especies), líquenes ( 2 especies), hongos (1 especie) y suelo acumulado en pequeños intersticios (1 especie) (Cuadro 1). A diferencia de los otros taxa, Trichia favoginea se observó principalmente sobre madera de albura.

Cuadro 1. Sustratos ocupados por las especies de mixomicetos en las Lagunas de Zempoala

\begin{tabular}{|c|c|c|c|c|c|c|}
\hline \multirow[b]{2}{*}{ Especie } & \multicolumn{6}{|c|}{ Sustratos } \\
\hline & $\begin{array}{l}\text { madera } \\
\mathrm{a}\end{array}$ & d & musgos & líquenes & hongos & suelo \\
\hline Ceratiomyxa fruticulosa & + & - & - & - & - & - \\
\hline Tubifera ferruginosa & - & + & + & - & - & - \\
\hline Lycogala epidendrum & + & + & - & - & - & + \\
\hline Enteridium lycoperdon & - & + & - & - & - & - \\
\hline Cribraria purpurea & - & + & - & - & - & - \\
\hline Dictydium cancellatum & - & + & + & + & - & - \\
\hline D. mirabile & - & + & - & - & - & - \\
\hline Arcyria cinerea & - & + & - & - & - & - \\
\hline A. ferruginea & - & + & - & - & - & - \\
\hline A. incarnata & - & + & - & - & - & - \\
\hline Hemitrichia clavata & - & + & + & - & - & - \\
\hline H. serpula & - & + & - & - & - & - \\
\hline Trichia decipiens & - & + & + & - & + & - \\
\hline T. favoginea & + & + & - & - & - & - \\
\hline Fuligo septica & - & + & - & - & - & - \\
\hline Physarum nutans & - & + & + & - & - & - \\
\hline Ph. viride & - & + & + & + & - & - \\
\hline Stemonitis axifera & - & + & - & - & + & - \\
\hline S. flavogenita & - & + & - & - & - & - \\
\hline Comatricha nigra & - & + & - & - & - & - \\
\hline
\end{tabular}


De acuerdo con los resultados obtenidos (Cuadro 1) y la bibliografía consultada, los mixomicetos hasta ahora encontrados en las Lagunas de Zempoala se pueden separar en tres grupos tomando en cuenta sus preferencias por determinados sustratos:

i) Especies que ocupan una amplia gama de sustratos (especies generalistas). En este grupo se incluyen Lycogala epidendrum, Arcyria cinerea, Hemitrichia clavata, Physarum nutans, Ph. viride y Fuligo septica.

ii) Especies lignicolas, es decir que sólo se han recolectado sobre diferentes clases de madera (duramen, albura, pequeñas ramas, tocones, etc.), incluyéndose aquí a Enteridium lycoperdon, Cribaria purpurea, Dictydium mirabile y Comatricha nigra.

iii) Especies lignicolas, pero que eventualmente pueden ocupar otros sustratos (suelo, musgos, líquenes, hojas, carpóforos de hongos, etc.). Aeste grupo corresponden Ceratiomyxa fruticulosa, Tubifera ferruginosa, Dictydium cancellatum, Arcyria ferruginea, A. incarnata, Hemitrichia serpula, Trichia decipiens, Stemonitis axifera y S. flavogenita.

En cuanto a la distribución de las especies, Martin y Alexopoulos (1969) consideraron como cosmopolitas a 15 de los 19 taxa aquí estudiados, en tanto Farr (1976) únicamente incluyó a 13 de estas especies en esta categorla. En ambos casos, se indico que Cribraria purpurea, Arcyria ferruginea, Hemitrichia clavata y Trichia favoginea presentaban una distribución más restringida. No obstante, las tres últimas han sido registradas de diversos ambientes tanto de zonas frías como templadas y tropicales (Cuadro 3), pero no de zonas áridas, en donde las condiciones ambientales parecen ser limitantes para su establecimiento. Estas tres especies junto con Enteridiumlycoperdon, Lycogala epidendrum, Tubifera ferruginosa, Dictydium cancellatum, Arcyria incarnata, Hemitrichia serpula, Trichia decipiens, Physarum nutans, Ph. viride, Stemonitis axifera, y S. flavogenita que también han sido citadas de zonas climáticas y ecológicas muy variadas, pero no de zonas áridas, pueden considerarse como organismos de amplia distribución, sin llegar a ser verdaderamente cosmopolitas.

Por otro lado, Ceratiomyxa fruticulosa, Arcyria cinerea, Fuligo septica y Comatricha nigra se han encontrado desde zonas tropicales húmedas y calientes, a zonas templadas y frías y regiones áridas y semiáridas (Cuadros 2 y 3), lo que sugiere que dichas especies sí podrían considerarse como cosmopolitas.

Cribraria purpurea y Dictydium mirabile solamente se conocen de lugares de clima templado en Europa, Norteamérica y la Unión Soviética (Cuadro 3), además de una colecta probable proveniente de una zona de clima tropical en Venezuela (Rodríguez, 1955, fide Farr, 1976) para la primera especie y un registro de Chile para $D$. mirabile (Farr, op. cit.). Hasta ahora, dichas especies se han registrado en México de dos localidades, ambas con una vegetación de bosque de confferas característica de zonas templadas (Cuadro 2).

En México la amplia distribución de algunas especies, como Tubifera ferruginosa, Dictydium cancellatum, Arcyria cinerea, Hemitrichia serpula y Fuligo septica, es muy clara, ya que se han recolectado en ambientes tan disímiles como los bosques tropicales, los mesófilos o los de coníferas (Cuadro 2). Otras, como Arcyria ferruginea y Comatricha nigra aparentemente tienen una distribución más restringida en nuestro país, sin embargo, su pequeño tamaño y la falta de estudios sistemáticos y exhaustivos en la mayor parte del territorio nacional parecen ser las causas que determinan el conocimiento sesgado de sus patrones de distribución. 
Galindo-Flores et al.: Los Mixomicetos del Parque Nacional Lagunas de Zempoala

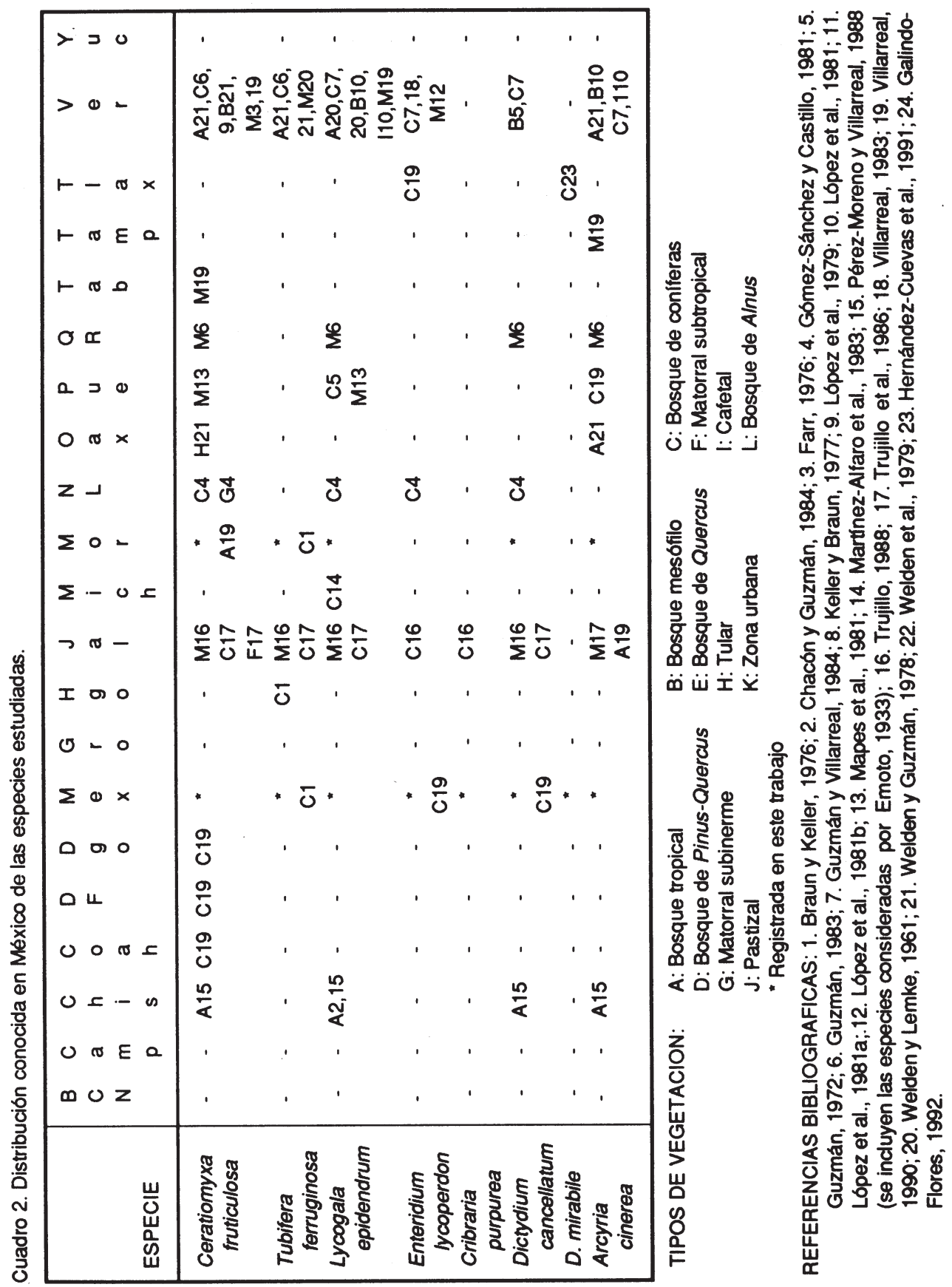




\begin{tabular}{|c|c|c|c|c|c|c|c|c|c|c|c|c|c|c|c|}
\hline$>$ & $\supset$ & 0 & & ' & ' & ' & & ' & $\sum$ & $\varphi$ & . & ' & $\frac{9}{\alpha}$ & & ' \\
\hline$>$ & 0 & 2 & & ' & 으 & ' & $\frac{N}{\frac{0}{0}}$ & ' & $\begin{array}{l}\hat{0} \\
\stackrel{0}{0}\end{array}$ & $\frac{N}{\frac{N}{N}}$ & ' & 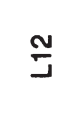 & ஸ્ฒ & হ̄ & ' \\
\hline$\vdash$ & - & ه & $x$ & రొ & . & ' & . & ' & ' & . & $\stackrel{ \pm}{y}$ & . & ' & . & . \\
\hline$\vdash$ & ه & $E$ & $a$ & ' & . & ' & . & , & ' & $\frac{9}{0}$ & . & , & . & ' & , \\
\hline$\vdash$ & & مـ & & ' & , & , & ' & , & ' & ' & . & ' & . & ' & . \\
\hline 0 & $\propto$ & & & ' & $\varphi$ & ' & $\stackrel{\varphi}{\Sigma}$ & ' & ' & $\xi$ & ' & 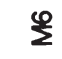 & $\frac{9}{2}$ & $\xi$ & ' \\
\hline a & ב & 0 & & ' & ! & ' & ' & ' & $\mathcal{O}$ & $\stackrel{m}{J}$ & \& & , & ' & ' & ' \\
\hline 0 & $\omega$ & $x$ & & ' & , & , & . & , & . & . & . & , & ' & ' & ' \\
\hline$z$ & \lrcorner & & & ' & ' & ' & J & J & ठ & 芒 & . & J & ' & ' & J \\
\hline$\Sigma$ & o & - & & , & * & , & , & * & * & . & , & ' & * & * & ' \\
\hline$\Sigma$ & - & 0 & ع & . & ' & ' & . & ' & . & $\frac{\pi}{0}$ & . & . & . & ' & ' \\
\hline$\supset$ & $\pi$ & - & & ' & ' & ' & $\hat{0}$ & ' & $\frac{0}{\infty}$ & $\stackrel{\oplus}{ \pm} \frac{N}{0}$ & . & ' & $\frac{0}{0} \frac{0}{0}$ & . & ' \\
\hline$I$ & б & $\circ$ & & ' & ' & ' & . & ' & . & . & , & ' & ' & ' & ' \\
\hline 0 & - & $\circ$ & & , & . & , & . & , & . & . & . & $\Xi$ & ' & . & . \\
\hline$\Sigma$ & 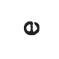 & $x$ & & * & ' & * & * & * & ' & * & * & * & * $\overline{0}$ & ' & * \\
\hline D & $\sigma$ & $\circ$ & & ' & . & . & . & ' & . & . & . & ' & ' & . & ' \\
\hline 0 & $\Perp$ & & & $\frac{0}{0}$ & ' & ' & ' & ' & . & , & . & ' & ' & ' & . \\
\hline 0 & o & ๘ & ع & . & . & . & ' & , & . & $\frac{O}{4}$ & , & ' & . & . & ' \\
\hline 0 & ع & - & os & . & $\frac{N}{<} \stackrel{n}{2}$ & $\frac{n}{4}$ & $\frac{n}{4}$ & , & . & ' & $\frac{n}{4}$ & . & ' & ' & ' \\
\hline 0 & గ & $\varepsilon$ & e & ' & ' & ' & $\frac{O}{<}$ & ' & ' & ' & ' & ' & , & ' & . \\
\hline$\infty$ & 0 & $z$ & & ' & ' & . & 1 & . & , & ' & . & . & $\frac{0}{0}$ & ' & . \\
\hline & & & $\begin{array}{l}\frac{w}{0} . \\
\text { w } \\
\frac{0}{w}\end{array}$ & 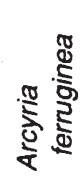 & 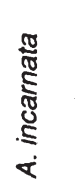 & 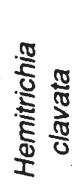 & $\begin{array}{l}\frac{\pi}{3} \\
\frac{2}{8} \\
\infty \\
I\end{array}$ & 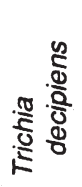 & 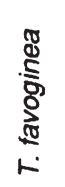 & : & 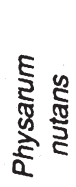 & $\begin{array}{l}\frac{0}{2} \\
\frac{10}{2} \\
\frac{1}{2}\end{array}$ & 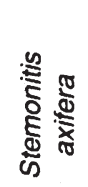 & 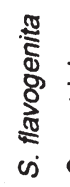 & 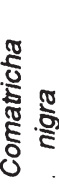 \\
\hline
\end{tabular}


Galindo-Flores et al.: Los Mixomicetos del Parque Nacional Lagunas de Zempoala

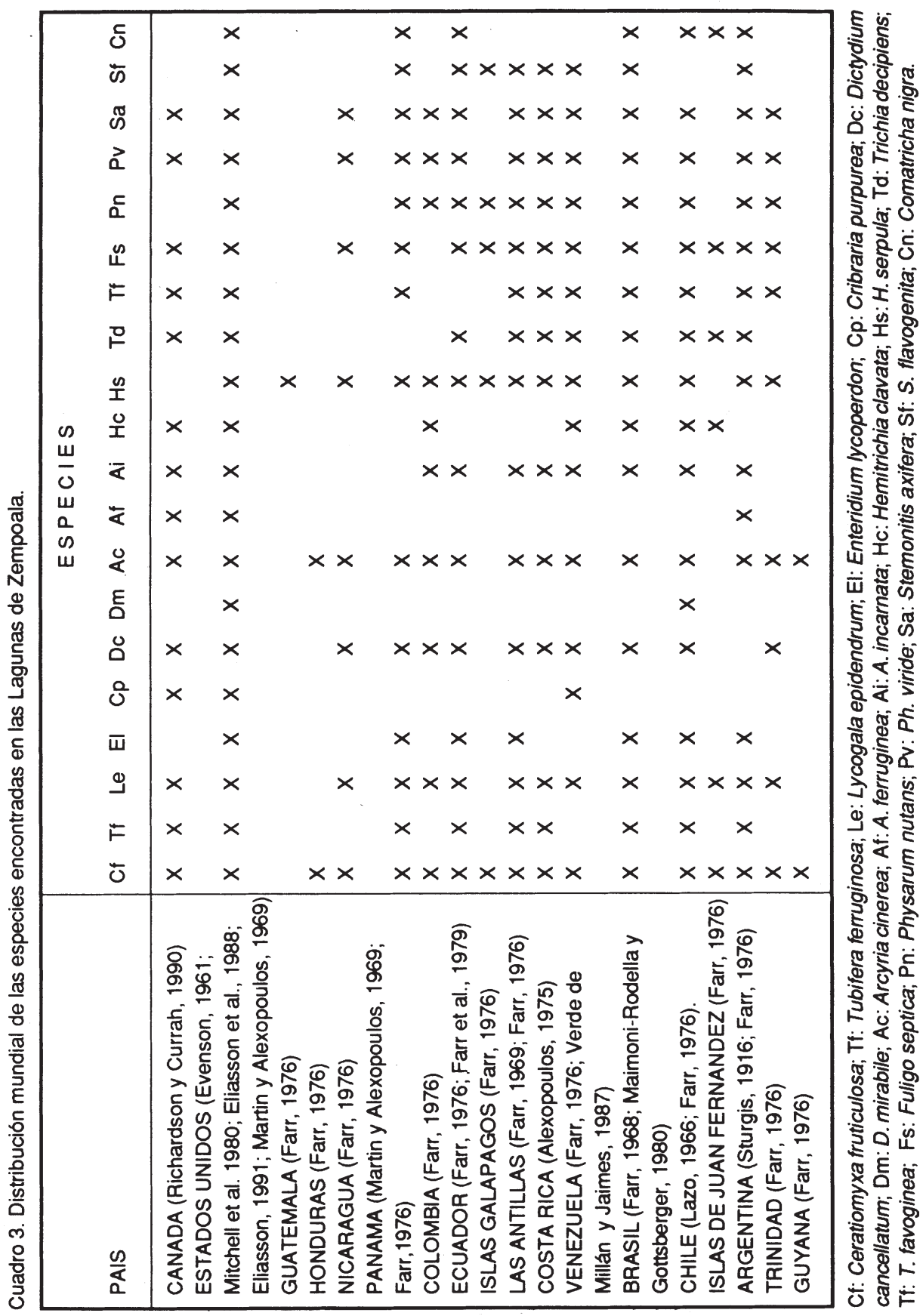




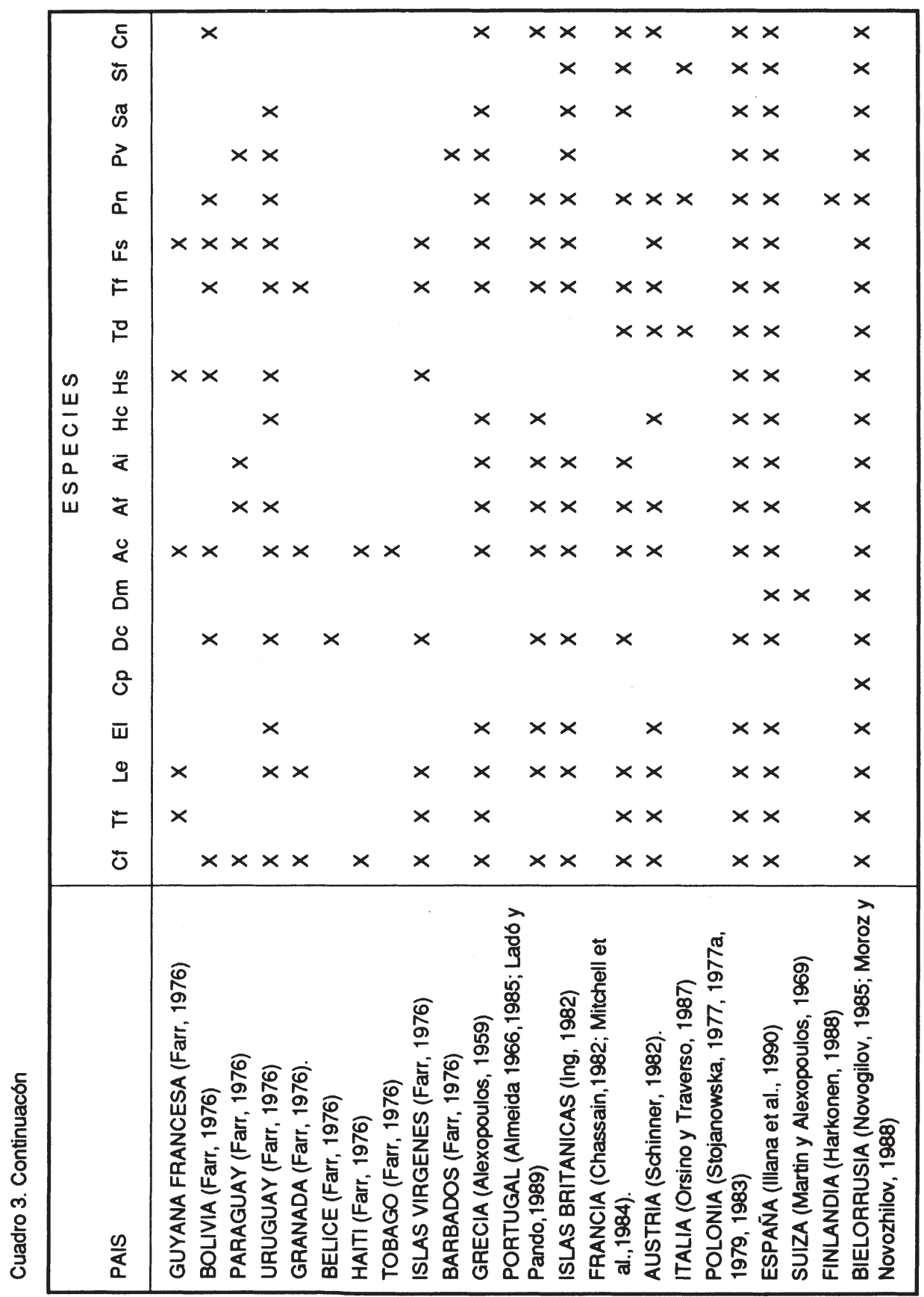


Galindo-Flores et al.: Los Mixomicetos del Parque Nacional Lagunas de Zempoala

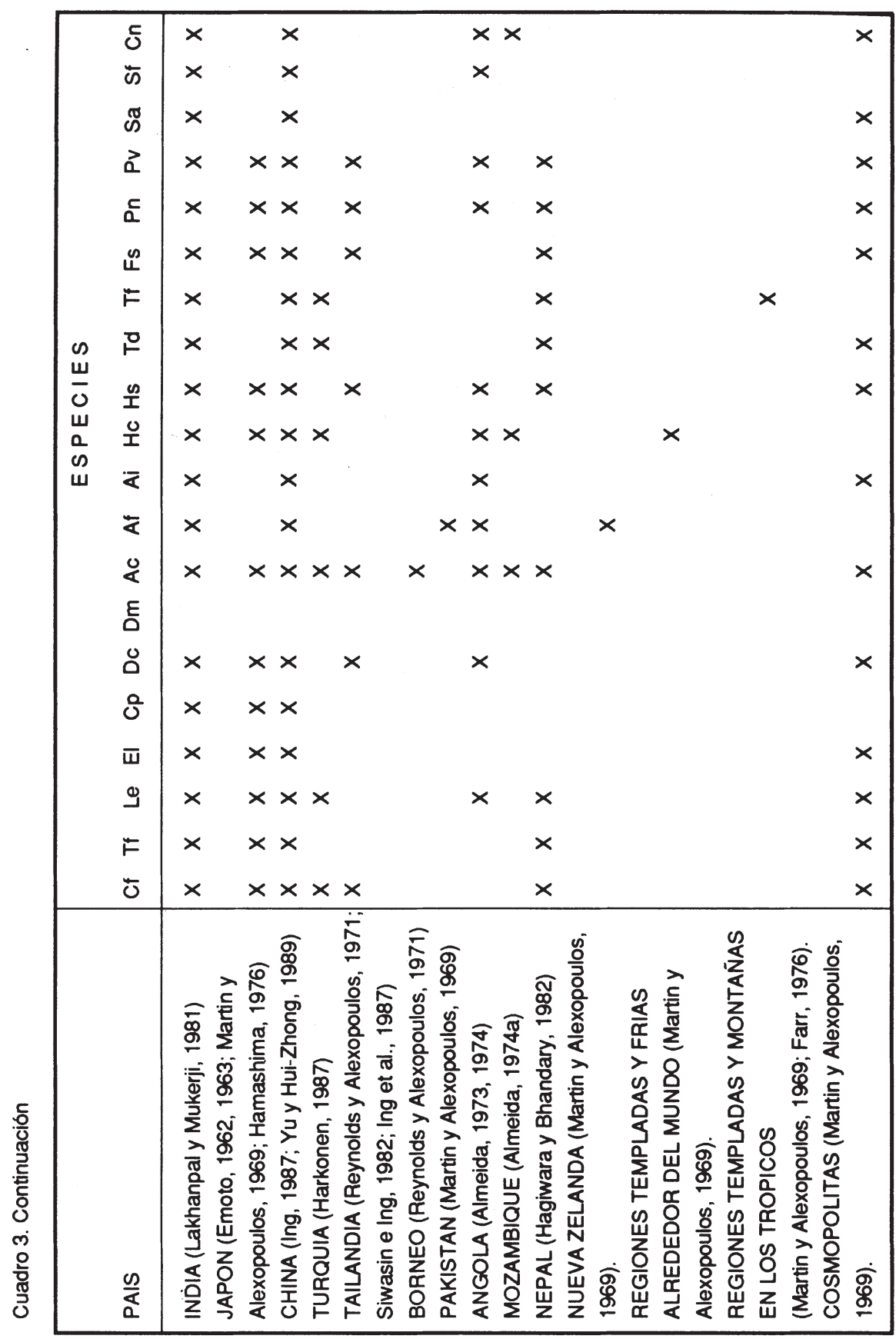




\section{AGRADECIMIENTOS}

Los autores expresan su agradecimiento a la M. en C. Elvira Aguirre, a la M. en C. Lucla Varela y al Dr. Miguel Ulloa por la revisión y sugerencias hechas al manuscrito.

\section{LITERATURA CITADA}

Alexopoulos, C. J. 1959. Myxomycetes from Greece. Brittonia 10(1): 25-40.

Alexopoulos, C. J. y A. Sáenz. 1975. The Myxomycetes of Costa Rica. Mycotaxon 2(2): 223-271.

Almeida, M. G. 1966. Contribuçáo para o estudo dos Myxomycetes de Portugal. II. Sep. da Rev. da Fac. de Ciencias de Lisboa, 2a Serie 14(1): 5-12.

Almeida, M. G. 1973. Contribuçâo para o conhecimento dos Myxomycetes de Angola. I. Bol. da Soc. Brot. $2 a$ Serie 47: 277-297.

Almeida, M. G. 1974. Contribuçâo para o conhecimento dos Myxomycetes de Angola. II. Bol. da Soc. Brot. 2a Serie 48: 187-203.

Almeida, M. G. 1974a. Contribuçâo para o conhecimento dos Myxomycetes de Moçambique. Bol. da Soc. Brot. 2a Serie 48: 205-210.

Almeida, M. G. 1985. Contribuçâo para o estudo dos Myxomycetes de Portugal. VII. Portug. Acta Biol.(B) 14: 5-10.

Braun, K. L. y H. W. Keller. 1976. Myxomycetes of Mexico I. Mycotaxon 3(2): 247-317.

Chacón, S. y G. Guzmán. 1984. Nuevas observaciones sobre los hongos, liquenes y mixomicetos de Chiapas. Bol. Soc. Mex. Mic. 19: 245-251.

Chassain, M. 1982. Essai sur L'écologie des Myxomycetes. Doc. Mycol. 12(46): 9-21.

Eliasson, U. 1991. Myxomycete biota of the Hawaiian islands. Mycol. Res. 95(3): 257-267.

Eliasson, U., H. W. Keller y J. A. Hutchinson. 1988. Myxomycetes from Arkansas. Mycotaxon 32: 375-398.

Emoto, Y. 1962. Myxomycetes found in Aichi-Prefecture. Trans. Mycol. Soc. Japan 3(1-6): 141-142.

Emoto, Y. 1963. List of Myxomycetes collected in the Masutomi Hot Spring Area, Yamanashi Pref., in August of 1960. Trans. Mycol. Soc. Japan 4(3): 2-4.

Evenson, A. E. 1961. A preliminary report of the Myxomycetes of Southern Arizona. Mycologia 53: 137-144.

Farr, M. L. 1968. An illustrated key to the Myxomycetes of South America, with special reference to Brazil. Rickia 3: 45-88.

Farr, M. L. 1969. Myxomycetes from Dominica. In: Smithsonian Institution (ed.). Bredin-Archbold-Smithsonian Biological Survey of Dominica. Smithsonian Institution Press, Washington.

Farr, M. L. 1976. Myxomycetes. In: New York Botanical Garden (ed.). Flora Neotropica 16. New York. 304 pp.

Farr, M. L. 1981. How to know the true slime molds. Wm. L. Brown, Dubuque, lowa. $131 \mathrm{pp}$.

Farr, M. L., U. Eliasson y K. P. Dumont. 1979. Myxomycetes from Ecuador. Mycotaxon 8(1): 127-134.

Frutis, I., R. E. Chio y A. Estrada-Torres. 1985. Nuevos registros de macromicetos del Estado de México. Rev. Mex. Mic. 1: 285-300.

Galindo-Flores, G. L. 1992. Algunos hongos del Jardín Botánico Tizatlán, Tlaxcala. Folleto No. 13. Talleres Gráficos del Estado de Tlaxcala, Tlaxcala. 62 pp.

García, E. 1988. Modificaciones al sistema de clasificación climática de Köppen. $5 a$ ed. México, D.F. 217 pp.

Gómez-Sánchez, A. y J. Castillo. 1981. Estudio sobre los mixomicetos del estado de Nuevo León. Bol. Soc. Mex. Mic. 15: 199-223.

Guzmán, G. 1972. Algunos macromicetos, líquenes y mixomicetos de la zona del Volcán Popocatépetl. In: Sociedad Botánica de México (ed.). Gulas botánicas de excursiones en México. I Congreso Latinoamericano de Botánica, México, D.F.

Guzmán, G. 1983. Los hongos de la península de Yucatán. II. Nuevas exploraciones y adiciones micológicas. Biotica 8(1): 71-100. 
Guzmán, G. y L. Villarreal. 1984. Estudio sobre los hongos, liquenes y mixomicetos del Cofre de Perote, Veracruz. I. Introducción a la micoflora de la región. Bol. Soc. Mex. Mic. 19: 107-120.

Hagiwara, H. y H. R. Bhandary. 1982. Myxomycetes from Central Nepal I. In: Reports on the Cryptogamic Study in Nepal (ed.). Miscellaneous Publication of the National Science Museum, Tokyo.

Hamashima, S. 1976. On the slime moulds found in the forest of Atsuta Shrine. Trans. Mycol. Soc. Japan 17: 88-89.

Härkönen, M. 1987. Some additions to the knowledge of Turkish Myxomycetes. Karstenia 27: 1-7.

Härkönen, M. 1988. Thirteen taxa of Myxomycetes new to Finland. Karstenia 28: 93-99.

Hernández-Cuevas, L., G. Galindo-Flores, M. Rodriguez-Palma y A. Estrada-Torres. 1991. New records of Myxomycetes from Mexico. Mycotaxon 42: 17-27.

IIlana, C., M. Heykoopy G. Moreno. 1990. Contribution to the study of the Myxomycetes in Spain. III. Catalogue of Myxomycetes of Spain. Mycotaxon 38: 37-69.

Ing, B. 1982. A revised census catalogue of British Myxomycetes. Part 2. Bull. of the Brit. Mycol. Soc. 16: 2636.

Ing, B. 1987. Myxomycetes from Hong-Kong and Southern China. Mycotaxon 30: 199-201.

Ing, B., J. Siwasin y S. Samarnpan. 1987. Myxomycetes from Thailand. II. Mycotaxon 30: 197.

Keller, H. W. y K. L. Braun. 1977. Myxomycetes of Mexico II. Bol. Soc. Mex. Mic. 19: 107-120.

Kornerup, A. y J. H. Wanscher. 1978. Methuen handbook of colour. Methuen. London. 252 pp.

Lado, C. y F. Pando. 1989. La colección de Myxomycetes preparada por C. Torrend para el Colegio San Fiel (Portugal). Anales Jard. Bot. Madrid 46(1): 173-180.

Lakhanpal, T. N. y K. G. Mukerji. 1981. Taxonomy of the Indian Myxomycetes. J. Cramer. Vaduz. 531 pp.

Lazo, W. R. 1966. Notes and illustrations of Myxomycetes from Chile and other countries. Mycologia 58: 6779.

López, A., A. Sosa y L. Villarreal. 1979. Estudio sobre los mixomicetos del estado de Veracruz I. Bol. Soc. Mex. Mic. 13: 127-144.

López, A., A. Sosa y L. Villarreal. 1981. Estudio sobre los mixomicetos del estado de Veracruz II. Biotica 6(1): 43-56.

López, A., L. Villarreal y A. Sosa. 1981a. Estudio sobre los mixomicetos del estado de Veracruz III. Bol. Soc. Mex. Mic. 16: 77-94.

López, A., L. Villarreal y A. Sosa. 1981b. Estudio sobre los mixomicetos del estado de Veracruz V. Bol. Soc. Mex. Mic. 16: 95-104.

López, L., V. M. Mora, E. Montiel y G. Guzmán. 1985. Nuevos registros de Agaricales del estado de Morelos. Rev. Mex. Mic. 1: 269-284.

Maimoni-Rodella, R. de C. y G. Gottsberger. 1980. Myxomycetes from the forest and the cerrado vegetation in Botucatu, Brazil: A comparative ecological study. Nova Hedwigia 34: 207-245.

Mapes, C., G. Guzmán y J. Caballero. 1981. Etnomicologia purépecha. El conocimiento y usos de los hongos en la Cuenca de Pátzcuaro. Secretaría de Educación Pública. Dirección General de Culturas Populares, Sociedad Mexicana de Micología y Universidad Nacional Autónoma de México. México, D.F. 79 pp.

Martin, G. W. y C. J. Alexopoulos. 1969. The Myxomycetes. University of lowa Press, lowa City. 559 pp.

Martínez-Alfaro, M. A., E. Pérez-Silva y E. Aguirre-Acosta. 1983. Etnomicología y exploraciones micológicas en la Sierra Norte de Puebla. Bol. Soc. Mex. Mic. 18: 51-63.

Mendiola, G. y G. Guzmán. 1973. Las especies de Tremellales conocidas de México. Bol. Soc. Mex. Mic. 7: 89-97.

Mitchell, D. H., S. W. Chapman y M. L. Farr. 1980. Notes on Colorado fungi. IV. Myxomycetes. Mycotaxon 10(2): 299-349.

Mitchell, D. H., E. Nannenga-Bremekamp, C. L. Champion y M. C. Clark. 1984. Myxomycota ramassés en France et conservés dans nos collections privés. Doc. Mycol. 14(54-55): 51-59.

Moreno, G., C. Illana y M. Heykoop. 1989. Contribution to the study of the Myxomycetes in Spain I. Mycotaxon 34(2): 623-635.

Moroz, E. L. y J. K. Novozhilov. 1988. Conspectus Myxomycetum RSS Belorussiae.. Nov. Syst. Plant. non Vasc. 25: 92-97. 
Novogilov, K. J., 1985. Conspectus Myxomycetum in URSS Vigentium. Nov. Syst. Plant. non Vasc. 22: 137143.

Orsino, F. y M. Traverso. 1987. Nuovi mixomiceti della Liguria. Mic. Ital. 2: 3-7.

Pérez-Moreno, J. y L. Villarreal. 1988. Los hongos y Myxomycetes del estado de Chiapas, México. Estado actual de conocimiento y nuevos registros. Mic. Neotrop. Aplic. 1: 97-133.

Richardson, F. A. y R. S. Currah. 1990. Myxomycetes of Alberta. Mycotaxon 37: 363-378.

Reynolds, D. R. y C. J. Alexopoulos. 1971. Southeast Asian Myxomycetes. I. Thailand and Burma. Pacific Sc. 25(1): 33-38.

Rzedowski, J. 1978. Vegetación de México. Limusa, México, D.F. 431 pp.

Siwasin, J. y B. Ing. 1982. Myxomycetes from Thailand. Nord. J. Bot. 2: 369-370.

Schinner, F. 1982. Myxomycetes of the Gro Bglockner region (Hohe Tauern Mts., Austria). An ecological study. Z. Mykol. 48(1):165-170.

Stojanowska, W. 1977. Changes in slime mold flora in the reservation of Puszcza Snieznej Bialki. Acta Mycol. 13(1): 99-107.

Stojanowska, W. 1977a. Slime mold flora of the Sleza massif. Acta Mycol. 13(2): 245-256.

Stojanowska, W. 1979. Observations on the slime molds growing on the moulder beech wood. Acta Mycol. 15(1): 167-174.

Stojanowska, W. 1983. Myxomycetes of the Sudetes. I. Acta Mycol. 19(2): 207-243.

Sturgis, W. C. 1916. Myxomycetes from South America. Mycologia 8: 34-41.

Toledo, V. M. 1988. La diversidad biológica de México. Ciencia y Desarrollo 14(81): 17-30.

Trujillo, F. 1988. Contribución al conocimiento de los Myxomycetes de laSierra de Manantlán, Jalisco. Tiempos de Ciencia 12: 20-27.

Trujillo, F., M. M. Castañeda y L. Guzmán-Dávalos. 1986. Hóngos del estado de Jalisco VI. Los mixomicetos conocidos. Tiempos de Ciencia 5: 42-54.

U. S. Department of Agriculture. 1975. Munsell soil color chart. Kollmorgen Corporation, Baltimore. 17 pp.

Verde de Millán, L. y F. Jaimes. 1987. Contribución al conocimiento de los Myxomycetes de Venezuela. I. Estado Sucre. Bol. Soc. Micol. Madrid 11(2): 195-201.

Villarreal, L. 1983. Algunas especies de Myxomycetes no registradas del estado de Veracruz. Bol. Soc. Mex. Mic. 118: 153-164.

Villarreal, L. 1990. Estudios sobre los Myxomycetes de México, I.Nuevos registros. Micol. Neotrop. Aplic. 3: 67-79.

Welden, A. L. y P. A. Lemke. 1961. Notas sobre algunos hongos mexicanos. Bol. Soc. Bot. Mex. 26: 1-23.

Welden, A. L. y G. Guzmán. 1978. Lista preliminar de los hongos, líquenes y mixomicetos de las regiones de Uxpanapa, Coatzacoalcos, Los Tuxtlas, Papaloapan y Jalapa (parte de los estados de Veracruz y Oaxaca). Bol. Soc. Mex. Mic. 12: 59-102.

Welden, A. L., L. Guzmán-Dávalos y G. Guzmán. 1979. Segunda lista de los hongos, líquenes y mixomicetos de las regiones de Uxpanapa, Coatzacoalcos, Los Tuxtlas, Papaloapan y Jalapa (México). Bol. Soc. Mex. Mic. 13: 151-161.

Yu, L. y L. Hui-Zhong. 1989. Myxomycetes from China I. A checklist of Myxomycetes from China. Mycotaxon 35(2): 429-436. 